\title{
Los fonemas, las sílabas y los morfemas como unidades de procesamiento ortográfico en el proceso lector de alumnos de Educación Infantil
}

\author{
Phonemes, syllables and morphemes as orthographic processing units in the process reader \\ kindergarten students
}

\author{
Pilar Vieiro-Iglesias, Ángela Fernández-Garrido, Mª Luisa Gómez-Taibo y Teresa García-Real \\ Universidade da Coruña
}

\begin{abstract}
Resumen
Analizamos la influencia de los fonemas, sílabas y morfemas en etapa infantil como condicionantes en el acceso al léxico ortográfico. Participó una muestra de 16 sujetos procedentes de una clase de $3^{\circ}$ de Educación Infantil, todos ellos lectores iniciados. Para determinar si poseían un nivel adecuado de elocución y comprensión del lenguaje, aspectos necesarios para formar parte del estudio, se les aplicó la prueba ELCE, y posteriormente las pruebas PSL y BLOC, para medir el conocimiento y el uso de fonemas, sílabas y morfemas dentro de la palabra. Se llevó a cabo un estudio de tipo correlacional, donde se encontró una correlación significativa entre las variables de morfosintaxis y las de fonología, y una correlación positiva entre las distintas variables de fonología y las de morfología. Así, la principal conclusión hallada en este estudio es que las unidades subléxicas se presentan como habilidades previas al acceso de la lectoescritura (acceso al léxico ortográfico) favoreciendo a su vez el aprendizaje y consolidación de los aspectos lectoescritores en esta etapa.
\end{abstract}

Palabras clave: léxico, morfosintaxis, fonología, conciencia silábica, lectura, Educación Infantil.

\footnotetext{
Abstract

The aim of this research was to study the influence of phonemes, syllables and morphemes in infancy as conditions in access to orthographic lexicon. To this end, we selected a sample of 16 subjects from a class of 3rd Child Education, all readers started. To determine if they had an adequate level of elocution and language comprehension aspects needed to be part of the study, we applied the test ELCE, and then the PSL and BLOC tests to measure knowledge and use of phonemes, syllables and morphemes within the word. Correlational study where a significant correlation between the variables of morphosyntax and phonology, and a positive correlation between the different variables of phonology and morphology was found out. Thus, the main conclusion found in this study is that sublexical units are presented as pre-literacy access (access to the orthographic lexicon) while promoting learning and consolidation of reader aspects at this stage skills.
}

Keywords: lexicon, morphosyntax, phonology, syllabic awareness , reading, kindergarten .

La investigación sobre el acceso al léxico visual ha demostrado que dicho reconocimiento no se reduce a una mera asociación entre estímulo y su representación mental; sino que es necesario algún tipo de análisis subléxico del estímulo para poder acceder al significado de la palabra (Taft y Forster, 1976; Tousman e Inhoff, 1992; Peressotti, Mulatti y Job, 2010).

De este modo, distintas operaciones de bajo nivel favorecerán el procesamiento de la palabra completa:

- procesamiento de los rasgos de las letras,

- su reconocimiento,

- conversión grafema-fonema,

- su representación léxica visual al convertir fonemas a grafemas,

- activación del significado de la palabra. (Marcolini, Burani y Colombo, 2009; Luque y López- Zamora, 2011; Simon, Lewis y Marantz, 2012).

Pero existen una serie de variables o parámetros lingüísticos que influyen en el reconocimiento de la palabra (Álvarez et al.,1995; Conrad, Carreiras, Tamm y Jacobs, 2009; Chetail y Mathey, 2010; Davies, Rodríguez-Ferreiro, Suárez y Cuetos, 2013; Defior, 1993; Harm y Seidenberg, 2004; de Vega et al.,1990, entre otros), tales como:

a) la longitud de la palabra;

b) la frecuencia léxica o índice de familiaridad de una palabra;

c) la lexicalidad o reconocimiento de cadenas de letras; d) la frecuencia grafémica o frecuencia posicional de letras, bigramas o sílabas dentro de la palabra;

y e) el procesamiento léxico por medio de la información visual, de la que posteriormente se establecerá una representación mental que servirá para el reconocimiento de la palabra.

Y en este contexto han sido muy pocos los estudios que relacionen estrechamente el papel de la sílaba en el ámbito de la producción escrita de palabras en lenguas semitransparentes como la nuestra. La literatura científica, escasa en nuestra lengua pero abundante en lenguas opacas, muestra evidencias procedentes de distintos campos que parecen determinar la realidad psicológica de la sílaba, el morfema y el fonema en el 
proceso de escritura en lenguajes de estructuras silábicas claras.

Los morfemas son las unidades más pequeñas dotadas de significado que representan unidades fonológicas, ortográficas, sintácticas y semánticas, y facilitan tanto el proceso de lectura de palabras como la comprensión de éstas en textos escritos (Bowers, Kirby y Deacon, 2010; Quemart, Casalis y Cole, 2011).

Elbro y Arnbak (1996), Taft y Forster (1976) y SánchezCasas (1996), indican que la frecuencia del morfema raíz de una palabra, produce un efecto de facilitación en el reconocimiento de la palabra, que haría más sencillo para el sujeto que se inicia en el proceso lectoescritor el acceso a la lectura y a la escritura, mantiene que ésta puede mejorar independientemente de la conciencia fonémica. Unas habilidades morfológicas bien desarrolladas sirven como cimientos sólidos sobre los que se construirá y apoyará la conciencia fonológica.

Si nos ceñimos estrictamente al papel ejercido por los morfemas en el ámbito de la escritura, cabe señalar que algunos experimentos determinan que la información morfológica es relevante, y se lleva a cabo su procesamiento mientras se está escribiendo.

Los fonemas son importantes, sobre todo, en los primeros años de aprendizaje ya que el aprendizaje de la lectura implica una conversión del lenguaje hablado al sistema escrito. De este modo, se establece que la relación entre conciencia fonológica y lectoescritura es bidireccional, puesto que la conciencia fonológica favorece la adquisición de la lectoescritura y su enseñanza desarrolla dicha conciencia.

La combinación de fonemas unida a la sonoridad del lenguaje proporciona a los lectores la llamada conciencia silábica fundamental en tareas básicas como la rima y la aliteración.

En este contexto el presente trabajo se propone examinar el desarrollo de las habilidades subléxicas tienen en el acceso al léxico en lectores principiantes pertenecientes al último curso de Educación Infantil, igualados en nivel de desarrollo del habla y de la comprensión verbal, considerados como prerrequisitos del aprendizaje lectoescritor según el modelo continuo de Bradley y Bryant (1983).

En este sentido nuestras hipótesis de trabajo plantearían el alto poder predictivo de las habilidades previas de lectoescritura a nivel subléxico (morfemas, sílabas y fonemas) en el acceso a la lectura así como un desarrollo paralelo e interrelacionado de las mismas en esta etapa evolutiva.

Con este fin llevamos a cabo el siguiente estudio en que analizaremos descriptivamente el nivel de eficacia de las distintas medidas utilizadas así como la interrelación entre ellas en una muestra de alumnos/as de $3^{\circ}$ de Educación Infantil igualados en nivel de vocabulario y desarrollo del lenguaje oral medidos a través del ELCE. Al análisis del perfil subléxico de la muestra va dirigido este trabajo.

\section{Participantes}

La muestra inicial de este trabajo estaba formada por 17 participantes $(n=17)$ castellano parlantes, con una edad media de 5.4 años. La muestra estaba formada por un $58.2 \%$ de niñas y un $41.8 \%$ de niños. Todos los participantes pertenecen a una misma clase, y se encuentran en la etapa de $3^{\circ}$ de Educación Infantil. Están escolarizados en un centro público en una zona con un nivel sociocultural medio-bajo. Ninguno de ellos muestra ningún problema específico en el lenguaje oral, así como ningún tipo de trastorno en el desarrollo o dificultad específica de aprendizaje.

Para su inclusión en el estudio se establecieron los siguientes criterios: a) que estuvieran iniciados en el aprendizaje de la lectoescritura y b) que presentasen un nivel óptimo en cuanto al desarrollo del habla y del lenguaje como prerrequisitos para el aprendizaje de la lectoescritura; para ello utilizamos la prueba ELCE, y dentro de ella seleccionamos las subescalas de Elocución y Comprensión verbal.

De los 17 sujetos a los que se les aplicó la prueba ELCE, 16 alcanzaron niveles óptimos de eficacia en su consecución; no obstante, 1 alumno fue eliminado de la muestra por no cumplir los requisitos, al tratarse de un sujeto prelector, y al evidenciar una baja ejecución en ELCE.

Las conclusiones que pudieron extraerse de la presentación inicial de la prueba ELCE, permitieron el establecimiento de un perfil general común a todos los participantes. Así, los sujetos objeto de estudio, mostraron una alta puntuación en comprensión verbal, con un buen manejo del lenguaje y unos niveles óptimos de comprensión en lo relativo al aspecto semántico. Inclusive el sujeto que fue posteriormente eliminado de la muestra inicial, mostró una comprensión adecuada del lenguaje. Fue sin embargo, en el apartado encargado de medir la Elocución y la Discriminación Auditiva, donde se detectaron algunas dificultades adicionales, motivadas en gran medida por el desconocimiento de vocabulario asociado a las imágenes que apoyaban esta prueba. No obstante, todos los alumnos presentaron una pronunciación fonémica notable y un uso del lenguaje adecuado, aspectos que pudieron inferirse del contacto establecido con ellos durante las sesiones de trabajo.

\section{Materiales}

Para la evaluación inicial del nivel de desarrollo de habilidades previas se utilizó como instrumento de medida la prueba ELCE.

La prueba ELCE de López Ginés et al. (2007), se basa en la exploración del habla y del lenguaje en el castellano a nivel de comprensión verbal y elocución.

A nivel de comprensión del lenguaje se evalúan el nivel sensorioperceptivo y el verbal puro, en los cuales se analizan tres aspectos: semántico, analítico-sintético y de pensamiento. Para este estudio nos hemos centrado en la medición del aspecto semántico, seleccionando para este fin el Test de Madurez de Metropolitan Item.

Esta prueba puede aplicarse a niños de entre 4 y 7 años de edad, por lo que se ajusta al rango de edad objeto de estudio. Incluye 14 láminas con 4 ilustraciones cada una, de entre las cuales el niño tendrá que seleccionar un ítem correcto para cada lámina. Estas ilustraciones irán agrupadas por categorías en función de su similitud semántica. 
La subescala de elocución tiene en cuenta la manera en que el niño hace uso de la palabra para expresar conceptos. En la elocución del lenguaje se valoran las capacidades fonoarticulatorias y las praxias, la exploración fonológica y fonética, la percepción y discriminación auditiva. Centrándonos en este último apartado hemos prestado atención a la discriminación fonética de los sujetos, para lo que hemos utilizado la prueba de Discriminación Fonética. Esta prueba valora si el niño discrimina los fonemas vocálicos y consonánticos en oposición de rasgos, según punto de articulación, modo, ambos y otros rasgos. Esta prueba consta de 10 láminas en las cuales aparecen cuatro dibujos para cada par de fonemas consonánticos opuestos. Los niños deben señalar cada uno de los dibujos y decir qué son, emitiendo correctamente los fonemas diferenciadores.

En cuanto a la evaluación de las unidades subléxicas, se ha llevado a cabo utilizando los instrumentos: Prueba de Segmentación Lingüística (PSL) de Jiménez y Ortiz (1998) y la Batería del Lenguaje Objetiva y Criterial de Puyuelo et al.(1997).

La prueba PSL consta de siete tareas: a) segmentación léxica donde el alumno debe reconocer el número de palabras que componen cada oración, pudiendo ayudarse para el recuento de los dedos de las manos, palmadas u objetos físicos manipulables; b) aislar fonemas o sílabas en las palabras en la que se han de identificar un fonema vocálico o consonántico y una sílaba en posición inicial y final; c) omisión de fonemas en las palabras (fonema vocálico inicial, sílaba inicial y sílaba final); d) reconocer si la sílaba inicial, media y final coincida con la de otra palabra en pares de palabras bisílabas y trisílabas; e) contar las sílabas en una palabra; freconocer y pronunciar la palabra descompuesta en una secuencia de sílabas donde el niño deberá ser capaz de reconocer cual es la palabra original tras haberla escuchado segmentada silábicamente; g) omisión de sílabas en las palabras en posición inicial o final en palabras bisílabas o trisílabas.

La Batería del Lenguaje Objetiva y Criterial de Puyuelo et al. (1997) es una prueba que se compone de cuatro grandes módulos. Cada uno de ellos se encarga de un componente diferente del lenguaje (morfología, sintaxis, semántica y pragmática). A la vez, cada uno de estos módulos se subdivide en una serie de bloques destinados a evaluar diferentes habilidades lingüísticas que se encuentran presentes en cada componente del lenguaje.

Para este trabajo únicamente se utilizó el módulo destinado al estudio de la morfología. La prueba de morfología se organiza en 19 bloques de contenido, donde se contemplan diferentes aspectos del lenguaje a tener en cuenta.

Se seleccionaron los módulos 1 (plurales de singulares acabados en vocal), 2 (plurales de singulares acabados en consonante y de singulares variables), 3 (adjetivos) y 12 (comparativos y superlativos).

\section{Diseño}

Llevamos a cabo un estudio de tipo correlacional que nos permitió analizar el nivel predictivo de las distintas habilidades objeto de estudio. En este sentido, las variables dependientes fueron el nivel morfológico medido a través de distintas puntuaciones del BLOC, y el nivel de Conciencia Fonológica medido a través de la prueba PSL.

\section{Procedimiento}

La aplicación de las pruebas se realizó de manera individual, escogiéndose los viernes como día para la realización de las sesiones de recogida de datos, siempre en horario lectivo. Fue necesaria previa autorización del centro educativo, que dio su consentimiento para la recogida de información.

Cada sesión individual tuvo una duración estimada de 45 minutos, en los que se trasladó a cada sujeto individualmente a un aula separada, para la aplicación de las tres pruebas consecutivamente; se aleatorizó la presentación de las mismas con el fin de evitar un posible sobreaprendizaje. El número de sesiones necesarias para la toma de datos correspondió a 17.

Para la realización de las pruebas se le dio a los sujetos la misma información relativa a la consecución de las actividades que debían realizar siguiendo el manual de instrucciones da cada una de las pruebas utilizadas.

Del análisis de los resultados en las distintas subtareas destaca el hecho de que todos los sujetos evaluados fuesen capaces de resolver adecuadamente todos los ejercicios planteados por la prueba BLOC de morfología, demostrando un adecuado conocimiento y aplicación de los morfemas en los subapartados incluidos en la prueba. De esta manera, el porcentaje de aciertos general para la Batería del Lenguaje Objetiva y Criterial fue del 100\%.

Atendiendo a la prueba PSL, los resultados fueron menos equilibrados entre los sujetos objeto de estudio. Se observan altos porcentajes generales de aciertos en los subapartados incluidos en la "Prueba de Segmentación Lingüística”, donde la mayor cantidad de errores puede observarse en el apartado relativo a la Omisión de la primera letra, cuyo porcentaje de la media de aciertos es notablemente inferior al resto de las pruebas (74\%), aunque debe insistirse en que los valores generales alcanzados por los niños fueron muy positivos y el índice de aciertos muy elevado. También encontraron dificultades en el ejercicio relativo a Contar las sílabas de una palabra, cuyo porcentaje de aciertos $(89,4 \%)$ es ligeramente inferior al porcentaje medio de aciertos en la prueba PSL que se situó en un 93,6\%. Los valores en Segmentación Léxica y Reconocimiento y pronunciación de una palabra descompuesta en una secuencia silábica se situaron en un $100 \%$ de aciertos, mostrando que la ejecución de los sujetos evaluados en esta prueba fue óptima. En el resto de los subapartados, los porcentajes alcanzaron valores por encima del $90 \%$, oscilando entre el $92,5 \%$ y el $97 \%$, evidenciando que, por lo general, la 
conciencia fonémica y silábica de los niños ha alcanzado valores muy positivos.

Con el fin de comprobar si efectivamente el conocimiento de las unidades subléxicas implica distintas habilidades o si por el contrario representa una única habilidad, relacionada con el acceso al léxico, calculamos la correlación entre las mismas a través de la prueba de Pearson Los resultados fueron significativos a un nivel $\mathrm{p}<.01$.

Posteriormente, se correlacionaron las puntuaciones de las distintas medidas de fonología. Los resultados fueron todos significativos. Para ello se expresaron las variables incluidas en la prueba con un valor numérico del 1-12, coincidiendo con el número de apartados medidos en el PSL. En algunos casos, la correlación hallada fue perfecta, es decir, se da el caso de una determinación absoluta entre las dos variables correlacionadas. Esto es en el caso de las variables 1 y 10, correspondientes a la Segmentación Léxica y el Reconocimiento y la pronunciación de la palabra descompuesta en una secuencia silábica. Se halló también que esta última variable mencionada, correlacionó también de manera perfecta con la variable 2, relativa al Aislamiento de sílabas y fonemas al inicio de palabra. Asimismo, la variable 3, centrada en Aislar sílabas y fonemas al final de la palabra, correlacionó de manera perfecta con las variables 11 y 12, esto es, las encargadas de Omitir las sílabas /ma/ y /bo/ en una palabra. También la variable 4, de Omisión de la primera sílaba de una palabra, halló un valor perfecto de correlación con las variables 11 y 12. En el resto de variables, se hallaron altos valores de correlación positiva, siendo esta significativa en todos los casos.

Se evitaron los análisis con las medidas de morfología pues en todas ellas se obtuvo la máxima puntuación.

\section{Discusión y Conclusiones}

En cuanto a los resultados descriptivos que han podido inferirse de las pruebas realizadas, se han mostrado altos valores de manejo y aplicación de los fonemas, los morfemas y las sílabas en estos sujetos, que como se explicitó en los requisitos previos, mostraban ya cierto dominio de los aspectos lectoescritores.

Su manejo de los morfemas en la formación de los plurales, el uso del adjetivo y la aplicación de los grados comparativo y superlativo fue óptimo, ya que ninguno de los sujetos evaluados mostró ninguna dificultad para la consecución de los ejercicios propuestos, los cuales requerían de la adhesión de los morfemas adecuados para la formación de palabras en cada uno de los bloques de la prueba. Estos resultados van en consonancia con los encontrados en los estudios de Álvarez, de Vega y Carreiras (1998) o Lázaro (2010) que consideran que la conciencia de los morfemas contribuye de manera crucial a la adquisición de la lecto-escritura. Burani (2009) lejos de lo que se podía pensar de que la conciencia de los morfemas sólo sería decisiva en lenguas opacas matiza que, incluso en ortografías transparentes estas unidades de amplio tamaño (los morfemas) juegan un papel decisivo como prerrequisitos del acceso al léxico o en cualquier caso de la fluidez en la lectura.

En lo relativo al manejo de los fonemas y las sílabas, tal y como se derivó de la Prueba de Segmentación
Lingüística, los niños mostraron unos valores muy elevados de dominio en los aspectos relativos a la segmentación de una palabra en unidades menores, encontrando mayores dificultades para enunciar una pseudopalabra resultante de la omisión de la primera letra de la palabra presentada. Tuvieron muchas dificultades para segmentar silábicamente de manera adecuada una palabra con presencia de diptongo, aunque generalmente su nivel de segmentación silábica fue óptimo en el resto de los ejemplos presentados. De estos resultados pudo derivarse que los sujetos han interiorizado el uso y aplicación de los fonemas y las sílabas como componentes de la palabra, siendo capaces de omitir dichas unidades de la palabra original, segmentarla silábica $\mathrm{y}$ fonémicamente, contar $\mathrm{y}$ reconocer el número de elementos presentes en ella, y en resumen, entender que la palabra se compone de unidades menores que ayudan a darle forma y sentido.

En esta dirección los resultados de nuestro estudio son coincidentes con los aportados por Alegría (2006), Casillas y Goicoetxea (2007) entre otros.

Atendiendo a los resultados obtenidos por los análisis que se llevaron a cabo, se llegó a la conclusión de que, tal y como se había planteado inicialmente coexisten varias habilidades en el acceso al léxico, todas ellas con un importante papel a la hora de desarrollar plenamente las capacidades de lectoescritura de los individuos. El correcto desarrollo de algunas de ellas parece apuntar directamente a un desarrollo posterior de las demás, iniciándose un proceso en cadena que favorecería el acceso al léxico, necesitando las unas de las otras para establecer un marco adecuado que favorezca la consolidación de los procesos de lectoescritura.

Esta afirmación se deriva de las altas correlaciones positivas entre las distintas variables de fonología y las de morfología y en especial en los casos de variables entre las que se encontró una correlación perfecta. De esta forma, se entiende que la variable de Reconocimiento y la pronunciación de la palabra descompuesta en una secuencia silábica, muestra un desarrollo paralelo a la variable de Segmentación Léxica y a la capacidad para Aislar sílabas y fonemas al inicio de la palabra; a su vez, la variable asociada a Aislar sílabas y fonemas al final de la palabra mostró una correlación perfecta con las variables de Omitir la sílaba /ma/ y /bo/ dentro de una palabra; por lo tanto se muestra que la capacidad de Aislar sílabas y fonemas al final de una palabra muestra un desarrollo paralelo y sirve para apoyar y sustentar la capacidad de Omitir una serie de sílabas dadas aleatoriamente en una palabra, citando la pseudopalabra restante con acierto. De esta manera, se evidencia que todos estos aspectos, los cuales se han contemplado en la evaluación de la Prueba de Segmentación Lingüística, son necesarios para una correcta representación del Léxico fonológico, y por lo tanto para un adecuado acceso al léxico.

Una interpretación a dichos resultados puede encontrarse en el efecto modelador del habla que presentan dichas habilidades, tal y como ha mostrado el estudio de Castro (1998). Por su parte, González (2005) encontró una relación altamente significativa entre prosodia y morfosintaxis. 
A la luz de los resultados obtenidos podríamos concluir:

- Se necesita de la coexistencia de varias habilidades vinculadas al desarrollo del léxico visual tales como la conciencia de los morfemas, de las sílabas y de los fonemas, posiblemente todas ellas vincualadas a un óptimo desarrollo del lenguaje oral.

- Dentro de estas habilidades, a su vez, tienen lugar diversos procesos que se apoyan entre sí, y cuyo desarrollo es paralelo. Esto quiere decir que es importante un adecuado desarrollo de la Conciencia Fonológica del individuo, que le permita comprender que las palabras se constituyen de unidades menores, como la sílaba, el fonema y el morfema. De esta capacidad para entender que la palabra puede segmentarse en unidades más pequeñas, dependerá el desarrollo de la lectura y las posibles dificultades que puedan ir surgiendo en la adquisición de la lectoescritura.

\section{Referencias Bibliográficas}

Álvarez, P., Zola-Morgan, S. y Squire, L. R., (1995). Damage limited to the hipocampal región produces long-lasting memory impairment in monkeys. Journal of Neuroscience, 15, 37963807.

Bowers, P. N., Kirby, J. R. y Deacon, S. H. (2010). The Effects of Morphological Instruction on Literacy Skills: A Systematic Review of the Literature. Review of Educational Research, 80, 144-179.

Bradley, L. y Bryant, P. E (1983). Categorizing sounds and learning to read: a causal connection. Nature 301, 419-421.

Chetail, F., y Mathey, S. (2010). InfoSyll: A syllabary providing statistical information on phonological and orthographic syllables. Journal of Psycholinguistic Research, 39, 485-504.

Conrad, M., Carreiras, M., Tamm, S. y Jacobs, A.M. (2009). Syllables and bigrams: Orthographic redundancy and syllabic units affect visual word recognition at different processing levels. Journal of Experimental Psychology: Human Perception and Performance, 35, 461-479.

Davies, R., Rodríguez-Ferreiro, J., Suárez, P. y Cuetos, F. (2013). Lexical and sub-lexical effects on accuracy, reaction time and response duration: impaired and typical word and pseudoword reading in a transparent orthography. Reading and Writing, 26, 721-738.

De Vega, M., Carreiras, M., Gutierrez, M. y Alonso, M.L. (1990). Lectura y comprensión: una perspectiva cognitiva. Madrid. Alianza Editorial.

Defior, S. (1993). Las dificultades de la lectura: papel que juegan las deficiencias en el lenguaje. Comunicación, Lenguaje y Educación, 17, 3 13.

Elbro, C. y Arnbak, E. (1996). The role of morpheme recognition and morphological awareness in dyslexia. Annals of Dyslexia, 46, 209-240.

Forster, K.I. (1976). Accessing the mental lexicon. En R.J. Wales \& E. Walker (Eds.), New Approaches to Language Mechanisms. North-Holland, Amsterdam.
Harm, M. y Seidenberg, M.S. (2004). Computing the meanings of words in reading: Division of labor between visual and phonological processes. Psychological Review, 111, 662-720.

Hautala, J., Aro, M., Eklund, K., Lerkkanen, M.-K., y Lyytinen, H. (2013). The role of letters and syllables in typical and dysfluent reading in a transparent orthography. Reading and Writing: An Interdisciplinary Journal, 26 (6), 845-864.

Jiménez, J.E. (1997). A Reading-Level Design Study of Phonemic Processes Underlying Reading Disabilities in a Transparent Orthography. Reading and Writing: An Interdisciplinary Journal. 9, 23-40.

Jiménez, J. y Ortiz, R. (1998). Prueba de Segmentación Lingüística. Madrid: TEA.

López Ginés, M.J.; Redón Díaz, A.; Zurita Calellas, M.D.; García Martínez, I.; Santamaría Mari, M. e Iniesta Matiarena, J. (2007). Exploración del lenguaje comprensivo y expresivo. ELCE. Madrid: Ciencias de la Educación Preescolar y Especial (4 ${ }^{a}$ edición).

Luque, D., Luque, J.L. y López-Zamora, M. (2011). Individual differences in pseudohomophony effect relates to auditory categorical perception skills. Learning and Individual Differences, 21, 210-214.

Marcolini, S., Burani, C., y Colombo, L. (2009). Lexical effects on children's pseudo word reading in a transparent orthography. Reading and Writing: An Interdisciplinary Journal, 22, 531-544.

Marslen-Wilson, W. D. (1987). Functional parallelism in spoken word-recognition. Cognition, 25, 71-102.

Peressotti, F., Mulatti, C. y Job, R. (2010). The development of lexical representations: Evidence from the position of the diverging letter effect. Journal of Experimental Child Psychology, 2, 177-183.

Puyuelo, M.; Renom, J. y Solanas, A. (1997). Manual de Evaluación Bloc. Batería de Lenguaje Objetiva y Criterial. Barcelona: Masson.

Quemart, P., Casalis, S., y Cole, P. (2011). The role of form and meaning in the processing of written morphology: A priming study in French developing readers. Journal of Experimental Child Psychology, 109, 478-496.

Sánchez-Casas, R. M. (1996). Lexical Access in Visual Word Recognition: the Contribution Word Form. En M. Carreiras, J.E. García-Albea y Sebastián Gallés, N. (eds.), Language Processing in Spanish. Hillsdale, NJ: Erlbaum.

Simon, D., Lewis, G. y Marantz, A. (2012). Disambiguating form and lexical frequency of MEG responses using homonyms. Language and Cognitive Processes, 27, 275-287.

Swinney, D. (1991). The resolution of indeterminancy during language comprehension: Perspectives on modularity in lexical, structural and pragmatic processing. En G.B. Simpson (Ed.), Understanding word and sentence, 367-385. Amsterdam: North-Holland. 
Taft, M. y Forster, K.I. (1976). Lexical storage and retrieval of polymorphemic and polysyllabic words. Journal of verbal Learning and verbal Behavior 15, 607-620.

Tousman, S. e Inhoff, A. (1992). Phonology in multisyllabic word recognition. Journal of Psycholinguistic Research, 21, 525-544.
Tunmer, W. E., y Chapman, J. W. (2012). The Simple View of Reading Redux:Vocabulary Knowledge and the Independent Components Hypothesis. Journal of Learning Disabilities, 45, 453-466. 